Annuaire suisse de politique de développement

25-1 | 2006

Faits et statistiques 2006

\title{
4. Aide humanitaire
}

\section{Gérard Perroulaz}

\section{OpenEdition}

Journals

Édition électronique

URL : http://journals.openedition.org/aspd/315

DOI : 10.4000/aspd.315

ISSN : 1663-9669

\section{Éditeur}

Institut de hautes études internationales et du développement

\section{Édition imprimée}

Date de publication : 1 avril 2006

Pagination : 47-59

ISBN : 2-88247-061-4

ISSN : $1660-5934$

Référence électronique

Gérard Perroulaz, «4. Aide humanitaire », Annuaire suisse de politique de développement [En ligne], 25-1 | 2006, mis en ligne le 10 février 2010, consulté le 07 septembre 2020. URL : http:// journals.openedition.org/aspd/315; DOI : https://doi.org/10.4000/aspd.315 


\section{Aide humanitaire*}

L 'AIDE HUMANITAIRE de la DDC s'est élevée à 281 millions de francs en 2004, attribués pour un tiers environ à l'aide internationale, un tiers au CICR et le reste pour l'aide bilatérale. La Confédération a développé ces dernières années certaines formes innovatrices d'aide humanitaire: aide au retour volontaire de réfugiés, aide financière directe aux personnes qui accueillent des victimes de catastrophes naturelles, approche en matière de réduction des risques de catastrophes (prévention).

L'actualité en 2004 et 2005 a été dominée par les nombreuses catastrophes naturelles meurtrières, notamment le tsunami en décembre 2004. L'ampleur de cette catastrophe a été importante, et la mobilisation des dons gouvernementaux et privés exceptionnelle. Si plusieurs facteurs peuvent expliquer les dons particulièrement élevés, la mobilisation n'est pas sans poser quelques questions, notamment la difficulté de mobiliser des dons pour des causes "oubliées», pour des catastrophes humanitaires moins médiatiques, ainsi que la difficulté d'assurer un financement à long terme.

Face à la succession de catastrophes naturelles, une importance grandissante est accordée aux actions de prévention, pour tenter de diminuer l'impact de ces catastrophes sur les populations menacées. C'est dans ce contexte qu'a eu lieu la Conférence mondiale de Kobe sur la prévention des catastrophes naturelles. Enfin, un emblème additionnel pour le Mouvement international de la CroixRouge et du Croissant-Rouge a été adopté en décembre 2005. Le "cristal rouge» s'ajoute ainsi à la croix rouge et au croissant rouge comme symboles universels du secours aux victimes des conflits armés.

L'aide humanitaire de la Confédération est régie par la Loi fédérale du 19 mars 1976 sur la coopération au développement et l'aide humanitaire ainsi que par son ordonnance d'application du 12 décembre 1977. Les montants alloués au titre de l'aide humanitaire sont puisés dans des crédits-cadres pluriannuels, pour lesquels le Conseil fédéral rédige un message concernant la continuation de l'aide humanitaire de la Confédération. Le dernier a été soumis au Parlement fédéral le 14 novembre $2001^{1}$. Ce crédit-cadre de 1,5 milliard de francs couvre la période de 2002 à 2006-2007.

C'est la Direction du développement et de la coopération (DDC) qui est en charge de la gestion de l'aide humanitaire de la Confédération (au Département fédéral des affaires étrangères DFAE), mais d'autres départements ou offices sont aussi concernés par l'aide d'urgence: le Département fédéral de la défense, de la protection de la population et des sports (DDPS) pour l'appui militaire aux opérations de secours, l'Office fédéral des migrations (ODM) pour l'aide au retour, la Division politique IV (Sécurité humaine). Les ONG suisses d'entraide

* Par Gérard Perroulaz, chargé de recherche à l'IUED.

1 Conseil fédéral, Message concernant la continuation de l'aide humanitaire internationale de la Confédération du 14 novembre 2001 (FF 2002 2087). 
jouent un rôle important dans l'aide humanitaire; les récoltes de fonds par la Chaîne du bonheur et par les diverses œuvres d'entraide suisses représentent une part non négligeable du «marché total du don» en Suisse. Les fonds récoltés pour les actions humanitaires sont recueillis principalement par les différentes ONG et par la Chaîne du bonheur. En 2004, celle-ci a récolté 38,9 millions de francs, dont 23 millions suite au tsunami (dons récoltés entre le 26 et le 31 décembre 2004), 7,3 millions suite au séisme à Bam (Iran), 4,1 millions lors des intempéries en Haïti (Caraïbes) et 2,1 millions pour l'aide au Darfour (Soudan)2 .

\subsection{Examen de l'aide humanitaire par le Comité d'aide au développement (CAD)}

Le rapport d'examen de la coopération suisse effectué par le CAD en 2005 relève la grande qualité de l'action humanitaire de la Suisse et le cadre juridique solide. La Suisse est l'un des rares pays où l'engagement humanitaire est ancré dans une loi $^{3}$. Plusieurs documents de base définissent les priorités de l'aide humanitaire. La stratégie 2005 de l'aide humanitaire précise les quatre missions prioritaires de l'aide humanitaire de la Confédération ${ }^{4}$ : la prévention, l'aide d'urgence, la reconstruction de logements et d'infrastructures de base et le plaidoyer $(\text { advocacy })^{5}$ en faveur des victimes et des exclus. L'aide humanitaire peut intervenir dans quatre types de situations: les conflits armés, les crises (effondrement des structures de l'Etat), les catastrophes naturelles et technologiques (par exemple Tchernobyl, pollution chimique).

L'augmentation des acteurs impliqués d'une manière ou d'une autre dans l'action humanitaire n'est pas sans poser des problèmes de coordination. L'examen de l'aide suisse par les pairs, effectué par le CAD en 2005, relève que les «doubles emplois et des positions contradictoires» peuvent survenir, notamment dans des situations d'urgence complexes. Autre exemple, la prévention des conflits est l'une des préoccupations de l'aide humanitaire, mais c'est aussi un domaine important au sein d'autres secteurs de la DDC. La Division politique IV du même département, le DFAE, chargée de la sécurité humaine, quant à elle, s'occupe d'un domaine proche avec la promotion de la paix (gestion civile des conflits $)^{6}$. Le rapport recommande un renforcement de la coordination entre les offices des différents départements.

Le rapport du CAD formule aussi les recommandations suivantes:

- la Suisse devrait parvenir progressivement à délier totalement l'aide alimentaire;

$\checkmark$ la DDC devrait concentrer davantage son soutien aux ONG suisses pour éviter une multiplication du nombre d'ONG dans la mise en œuvre de l'aide humanitaire;

2 Chaîne du bonheur, Rapport annuel 2004, 2005.

Loi fédérale de 1976 sur la coopération au développement et l'aide humanitaire internationales.

4 DDC, Vivre la solidarité. Aide humanitaire: stratégie 2005. Les domaines d'engagement généraux de l'aide humanitaire sont expliqués sur le site de la DDC, <www.ddc.admin.ch> >aide humanitaire $>$ domaines d'engagement.

5 SDC, Advocacy Guidelines: Humanitarian Aid of the Swiss Confederation, Bern, SDC, 2004.

6 CAD (OCDE), Examen par les pairs. Suisse. 2005, Paris, OCDE, 2005, pp. 65 et 113. 
- la Suisse devrait évaluer les coûts du déploiement rapide (du type de la Chaîne suisse de sauvetage) et les comparer au coût du renforcement des capacités locales ${ }^{7}$;

- la Suisse devrait évaluer les programmes d'aide au retour volontaire de réfugiés sous l'angle de leur efficacité et du respect des principes humanitaires.

\subsection{Aide humanitaire de la Suisse en 2004}

Tableau 7 : DDC: aide humanitaire, 2004 (en millions de francs)

\begin{tabular}{|c|c|c|c|c|}
\hline & $\begin{array}{l}\text { Contributions } \\
\text { générales }\end{array}$ & $\begin{array}{l}\text { Contributions } \\
\text { spécifiques }\end{array}$ & $\begin{array}{c}\text { Aide } \\
\text { alimentaire }\end{array}$ & Total \\
\hline Organisations internationales & $30.8^{\mathrm{a}}$ & 49.1 & 19.7 & 99.7 \\
\hline Programme alimentaire mondial (PAM) & 1.5 & 18.1 & 18.2 & 37.8 \\
\hline Haut-Commissariat des NU pour les réfugiés (HCR) & 13.5 & 16.4 & & 29.9 \\
\hline UNRWA & 12.7 & 1.3 & & 14.0 \\
\hline $\begin{array}{l}\text { Bureau de la coordination des affaires } \\
\text { humanitaires (OCHA) }\end{array}$ & 2.2 & 5.2 & & 7.5 \\
\hline Fonds des NU pour l'enfance (UNICEF) & & 2.8 & & 2.8 \\
\hline Autres organisations internationales & 0.9 & 5.2 & 1.5 & 7.7 \\
\hline Organisations de la Croix-Rouge internationale & 70.5 & 23.5 & & 93.9 \\
\hline Comité international de la Croix-Rouge (CICR) & 69.5 & 23.0 & & 92.4 \\
\hline $\begin{array}{l}\text { Fédération internationale des Sociétés de la } \\
\text { Croix-Rouge et du Croissant-Rouge }\end{array}$ & 1.0 & 0.5 & & 1.5 \\
\hline ONG suisses & & 18.9 & 12.0 & 30.8 \\
\hline $\begin{array}{l}\text { Actions directes du Corps suisse } \\
\text { d'aide humanitaire (CSA) }\end{array}$ & & 37.9 & 0.5 & 38.4 \\
\hline Autres actions d'aide humanitaire 2004 & & 16.9 & 1.1 & 18.0 \\
\hline Total aide humanitaire 2004 & 101.3 & 146.3 & 33.2 & 280.9 \\
\hline
\end{tabular}

Source: DDC/seco, Rapport annuel de la coopération internationale de la Suisse 2004, Berne, 2005.

a Ce montant représente l'aide humanitaire multilatérale de la DDC. Le reste relève de l'aide humanitaire bilatérale, soit 250 millions de francs, détaillés dans le tableau 8 plus bas.

L'aide humanitaire de la DDC s'est élevée à 281 millions de francs en 2004 et à 299,1 millions de francs en 2005 (chiffre pour 2005 provisoire). Le tableau 7 donne une vue d'ensemble de l'aide humanitaire de la DDC en 2004. Près de 100 millions de francs d'aide humanitaire ont été acheminés par le biais des organisations internationales. Les principales organisations internationales partenaires ont été le Programme alimentaire mondial, le Haut-Commissariat des Nations unies pour les réfugiés (HCR), l'Office de secours et de travaux des Nations unies pour les réfugiés de Palestine dans le Proche-Orient (UNRWA) et le Bureau de la coordination des affaires humanitaires des Nations unies (OCHA). Sont considérés comme de l'aide bilatérale les 94 millions de francs versés par le biais des organisations de la Croix-Rouge internationale. Les ONG suisses ont reçu 30,8 millions de francs de la DDC pour financer leurs actions humanitaires, de sorte que $11 \%$ de l'aide humanitaire de la DDC sont acheminés par l'intermédiaire des ONG suisses. Les principales ONG bénéficiaires de ces contributions ont été (par ordre décroissant) Caritas Suisse, Terre des hommes, la Croix-Rouge suisse, Médecins sans frontières, Medair et l'Armée du Salut. En 2004, les actions

7 Il n'est pas toujours facile de coordonner un nombre excessif d'équipes de recherche et de sauvegarde sur le terrain et les capacités locales de secours. 
directes du Corps suisse d'aide humanitaire (CSA) se sont élevées à 38,4 millions de francs ( $14 \%$ de l'aide humanitaire) et le CSA est intervenu dans 59 pays.

\subsubsection{Domaines d'activité de l'aide humanitaire en 2004}

Les instruments mis en œuvre dans le cadre de l'aide humanitaire sont l'envoi de personnel et de matériel (Corps suisse d'aide humanitaire), les contributions financières (aux organisations internationales et aux pays partenaires) et l'aide alimentaire. L'aide alimentaire s'est élevée à 33,2 millions de francs en 2004 (19 millions pour les produits laitiers suisses et 14 millions pour les céréales, souvent achetées localement ou dans les pays proches).

\subsubsection{Répartition géographique de l'aide humanitaire en 2004}

Tableau 8: DDC: aide humanitaire bilatérale, en 2004 et pour les trois années précédentes (en millions de francs)

\begin{tabular}{|c|c|c|c|c|}
\hline & 2004 & 2003 & 2002 & 2001 \\
\hline Afrique & 70.9 & 61.0 & 59.1 & 53.4 \\
\hline dont Soudan & 13.6 & 6.9 & 5.7 & 7.0 \\
\hline Angola & 7.6 & 7.8 & 8.2 & 7.1 \\
\hline Burundi & 4.6 & 3.0 & 3.7 & 6.6 \\
\hline Congo (RD) & 4.1 & 5.6 & 5.6 & 6.8 \\
\hline Liberia & 4.0 & 3.7 & 2.6 & 1.0 \\
\hline Tchad & 3.9 & 0.7 & 0.3 & 0 \\
\hline Sierra Leone & 3.6 & 3.5 & 4.5 & 5.7 \\
\hline Ouganda & 3.4 & 1.0 & 0.1 & 0 \\
\hline Ethiopie & 2.5 & 5.0 & 1.6 & 2.2 \\
\hline Asie & 45.8 & 49.3 & 49.3 & 43.8 \\
\hline dont Afghanistan & 12.0 & 14.5 & 16.9 & 10.8 \\
\hline Palestine & 5.8 & 5.5 & 2.1 & 1.6 \\
\hline Géorgie & 5.3 & 6.1 & 3.8 & 2.2 \\
\hline Sri Lanka & 3.0 & 2.8 & 3.2 & 0.1 \\
\hline Pakistan & 2.6 & 1.2 & 0.2 & 0.1 \\
\hline Irak & 1.8 & 6.0 & 3.8 & 4.8 \\
\hline Indonésie & 1.8 & 1.3 & 2.1 & 0.7 \\
\hline Europe & 19.1 & 23.4 & 64.2 & 28.1 \\
\hline dont Russie & 5.5 & 6.2 & 14.4 & 6.0 \\
\hline Moldova & 3.3 & 4.2 & 3.1 & 2.9 \\
\hline Serbie-Monténégro & 3.1 & 5.6 & 8.5 & 10.9 \\
\hline Amérique latine & 16.0 & 13.5 & 14.0 & 12.6 \\
\hline dont Colombie & 4.8 & 5.0 & 4.3 & 2.8 \\
\hline Pérou & 3.1 & 2.0 & 3.2 & 2.0 \\
\hline Haïti & 2.8 & 0.1 & 0.1 & 0.1 \\
\hline Brésil & 1.1 & 1.6 & 1.7 & 0.9 \\
\hline Cuba & 1.0 & 0.7 & 0.7 & 0.3 \\
\hline Nicaragua & 0.5 & 1.2 & 0.8 & 0.7 \\
\hline Non ventilé géographiquementa & 98.2 & 97.0 & 99.6 & 89.1 \\
\hline Total & $250^{\mathrm{b}}$ & 244.2 & 270.3 & 227.2 \\
\hline
\end{tabular}

Sources: DDC, Service statistique; DDC/seco, Rapport annuel de la coopération internationale de la Suisse, éditions 2002 à 2005.

a Comprend les contributions au Comité international de la Croix-Rouge (CICR) et à la Fédération internationale des Sociétés de la Croix-Rouge et du Croissant-Rouge.

b Ce total représente I'aide humanitaire bilatérale de la DDC aux pays en développement (239 millions de francs) et aux pays en transition (11 millions de francs). 
La répartition géographique de l'aide humanitaire bilatérale de ces dernières années est présentée dans le tableau 8. C'est le continent africain qui reçoit la part la plus importante de l'aide humanitaire de la DDC (28\%), suivi par le continent asiatique (18\%), l'Europe (pays en développement ou en transition, $7,6 \%$ ) et l'Amérique latine (6,4\%). $40 \%$ de l'aide ne peuvent pas être ventilés géographiquement (notamment les contributions au CICR). La part de l'aide humanitaire pour les pays de l'Europe est en baisse ces dernières années (elle représentait $24 \%$ de l'aide en 2002), les pays du Sud-Est de l'Europe étant dans une phase de reconstruction postconflit. Les données sur l'aide humanitaire dans les pays d'Asie touchés par le tsunami le 26 décembre 2004 n'apparaîtront que dans les statistiques 2005, car peu d'argent a été déboursé entre le 26 décembre et le 31 décembre 2004 (environ 2 millions d'aide humanitaire débloqués par la DDC pendant cet intervalle) (voir plus bas section 4.3.1).

\subsection{Principales actions d'aide humanitaire en 2004 et 2005}

\subsubsection{L'action humanitaire suite au tsunami (décembre 2004)}

Le tremblement de terre sous-marin et le tsunami du 26 décembre 2004 ont touché plusieurs pays d'Asie du Sud. Des montants records de récoltes de fonds privés auprès du public ont suivi cette catastrophe.

\section{$\square$ Actions de la Confédération (DDC)}

La DDC a engagé des fonds humanitaires à hauteur de 35 millions de francs (situation au 16 décembre 2005) pour l'aide humanitaire dans les pays sinistrés, dont 25,7 millions pour l'aide bilatérale et 9,3 millions pour l'aide multilatérale ${ }^{8}$. Les trois principaux pays d'intervention de l'aide suisse sont le Sri Lanka (16 millions de francs), l'Indonésie (13 millions) et la Thaïlande (4,4 millions). De petites contributions ont aussi été accordées à l'Inde (850'000 francs), aux Maldives, aux Seychelles et à la Somalie (un total de 600'000 francs pour ces trois pays).

L'une des formes originales de l'aide de la DDC est le programme «Cash pour familles d'accueil», qu'elle mène en étroite collaboration avec les œuvres d'entraide partenaires au Sri Lanka et en Indonésie. Il s'agit de donner des contributions financières (argent liquide) à des familles qui accueillent chez elles des personnes déplacées et des réfugiés ayant perdu leur logement ${ }^{9}$. Ce soutien a les avantages suivants:

$\checkmark$ aide pour des ménages privés pauvres qui accueillent des sans-abri chez eux;

- hébergement socialement plus digne que dans les grands camps de réfugiés, car il permet au bénéficiaire de surmonter plus facilement son statut de «victime» et de reprendre le contrôle de son avenir;

$\checkmark$ forme d'aide beaucoup moins coûteuse que la construction et la gestion de grands camps de réfugiés:

8 DDC, engagements de la DDC au 16 décembre 2005, sur le site Internet de la DDC, <www.ddc. admin.ch > >aide humanitaire >séisme et tsunami en Asie du Sud: une année après.

9 DDC, Cash pour familles d'accueil en faveur des victimes des raz-de-marée en Asie du Sud, fact sheet, s.d. 
$\square$ meilleure intégration sociale des orphelins ;

๖ augmentation du pouvoir d'achat dans l'économie locale.

Cette forme d'aide convenait aussi particulièrement bien aux caractéristiques des effets du tsunami, car elle s'insère dans la solidarité locale des habitants de l'intérieur des terres, peu touchées par la catastrophe, pour les habitants de la côte dévastée.

Ce genre de soutien à l' «hébergement de voisinage» est pratiqué par la DDC depuis les années 1990, surtout en Europe du Sud-Est, en Bosnie et au Kosovo, avec notamment des contributions financières en liquide pour des familles d'accueil ainsi que pour la réparation ou la reconstruction des habitations ${ }^{10}$. De 1999 à 2004, 17 millions de dollars ont été distribués ainsi dans le cadre de l'aide financière directe.

\section{Exemples de projets menés par la DDC dans les pays touchés par le tsunami}

Indonésie: soutien financier pour l'hébergement de sans-abri dans 7500 familles, remise en état de centrales hydrauliques, nettoyage de puits, soutien aux autorités indonésiennes pour la coordination des activités de reconstruction.

Sri Lanka: construction d'écoles provisoires et reconstruction de bâtiments scolaires, aide à la reconstruction de 8000 habitations.

Thaïlande: reconstruction de villages de pêcheurs (logements, écoles, voies de communication et débarcadères), construction d'une centaine de bateaux de pêche, reconstruction d'un centre de santé.

Inde: latrines et équipements sanitaires dans des camps de réfugiés.

Iles Maldives: programmes pour l'eau potable et l'hygiène communautaires.

Iles Seychelles et Somalie: versements de la DDC pour le PAM et la Fédération internationale de la Croix-Rouge et du Croissant-Rouge.

Source: site Internet de la DDC, <www.ddc.admin.ch> >aide humanitaire >séisme et tsunami en Asie du Sud: une année après, avec des fiches par pays pour les projets soutenus par l'aide humanitaire.

\section{$\square$ Ampleur sans précédent des récoltes de dons privés par la Chaîne du bonheur et les ONG suisses}

Les dons privés récoltés en Suisse et dans certains pays industrialisés suite à la catastrophe du tsunami ont été sans précédent. Fin juin 2005, les dons versés à la seule Chaîne du bonheur s'élevaient à 226 millions de francs, un montant record pour cette organisation ${ }^{11}$. Ce montant ne comprend pas les montants récoltés directement par les œuvres d'entraide suisses auprès de la population, mais constituera un financement très important des activités de ces œuvres d'entraide. Il ne couvre pas non plus l'aide directe issue de la forte mobilisation des

10 Les projets cash for shelter ont été développés dans les pays/régions suivantes: Albanie, Géorgie, Ingouchie (Fédération de Russie), Macédoine, Moldavie, Mongolie, Kosovo, Serbie. Voir: DDC, Projets cash pour l'aide humanitaire. Une forme novatrice de soutien direct, fact sheet, s.d.

11 Pour mémoire, les «records de dons » précédents de la Chaîne du bonheur avaient atteint 72 millions de francs pour les intempéries en Suisse (2000), 49 millions pour le Kosovo (1999), 34 millions suite à l'ouragan Mitch en Amérique centrale (1998), 14 millions suite au tremblement de terre en Inde (2001) et 14 millions suite au conflit au Sud-Soudan. Sources: Chaîne du Bonheur, rapports annuels, différentes années. 
communautés originaires des pays touchés qui résident en Suisse. La Chaîne du bonheur ne gère en effet pas elle-même les projets, mais finance les projets présentés par les organisations suisses d'entraide qui sont ses partenaires ${ }^{12}$ (29 organisations au 15 janvier 2005). Les ONG partenaires doivent fournir $10 \%$ de fonds propres récoltés par elles-mêmes. Plusieurs facteurs peuvent expliquer le montant record des dons privés récoltés par la Chaîne du bonheur: l'ampleur exceptionnelle de la catastrophe, qui a touché plusieurs pays, sa forte médiatisation pendant les fêtes de fin d'année, et les victimes européennes.

Une année après le tsunami, en décembre 2005, plus de 122 millions de francs étaient déjà engagés par la Chaîne du bonheur et la trentaine d'organisations partenaires pour aider les régions sinistrées, et les projets en cours d'examen représentaient un montant total de 34 millions de francs. Les fonds récoltés par la Chaîne du bonheur servent à financer les projets soumis par des ONG suisses spécialisées dans l'aide d'urgence, mais aussi par des ONG suisses habituellement concernées davantage par la coopération au développement à plus long terme (telles que Swisscontact par exemple), mais présentes dans les pays touchés par les conséquences du tsunami ${ }^{13}$. Les projets ont été menés principalement au Sri Lanka (projets en cours pour un montant de 46,3 millions de francs), en Indonésie (40,4 millions), en Inde (30,6 millions), en Thaïlande (2,9 millions) et en Somalie (1,8 million) (situation fin décembre 2005). 17\% des fonds sont consacrés à des projets d'aide d'urgence, et $83 \%$ à des projets de reconstruction et de réhabilitation.

La mobilisation des dons a été dans l'ensemble massive et l'aide totale promise en février 2005 dépassait les 13 milliards de dollars pour tous les pays donateurs (aide publique de gouvernements et aide privée) $)^{14}$. Pour les observateurs, l'ampleur énorme des dons privés témoignait de la capacité des gens à répondre aux appels de fonds pour des causes humanitaires, qui contraste avec une certaine retenue des parlementaires dans l'adoption de budgets de la coopération au développement et avec une aide publique au développement stagnante dans beaucoup de pays du Nord ${ }^{15}$.

L'ampleur de cette mobilisation soulève plusieurs questions ${ }^{16}$. Certaines d'entre elles sont liées à ce cas particulier d'intervention humanitaire, d'autres facteurs valent pour tout type d'intervention humanitaire. Ce sont des problèmes de coordination et d'acheminement de l'aide que l'ampleur exceptionnelle des dons a mis le plus fortement en évidence:

12 La Chaîne du bonheur a soutenu les actions humanitaires et de reconstruction des principales ONG suivantes: ADRA, ASED, Armée du Salut, Caritas Suisse, Croix-Rouge suisse, Entraide protestante, Frères de nos frères, Helvetas, Medair, OSEO, Swisscontact et Terre des hommes Lausanne. Elle a aussi versé de l'argent à des familles suisses touchées par le tsunami et au HCR.

13 Pour des informations sur ces projets, voir le site Internet de la Chaîne du bonheur, <www. bonheur.ch>, et la brochure de la Chaîne du bonheur Tsunami. Un an après (décembre 2005).

14 Propos de Jan Egeland, coordinateur des Nations unies pour les secours d'urgence, Le Temps, 14 janvier 2005.

15 «La tragédie asiatique réamorce le débat sur les coupes budgétaires de l'aide au développement», Le Temps, 7 janvier 2005; «Auslandhilfe zwischen Spenden und Sparen», Neue Zürcher Zeitung, 22.-23. Januar 2005.

16 De nombreux articles ont relevé les problèmes posés par cette récolte de dons exceptionnelle suite à une catastrophe très médiatisée. Par exemple «Les organisations humanitaires consentent à la dictature de l'image», Le Temps, 5 janvier 2005, 10 février 2005. 
$\square$ le problème de la capacité d'absorption de l'aide sur place ${ }^{17}$;

- le problème de la coordination sur place de l'aide d'une multitude d'acteurs gouvernementaux, multilatéraux et non gouvernementaux. Des centaines d'ONG sont intervenues au Sri Lanka et en Indonésie, dont la majorité n'étaient pas traditionnellement dans ces pays;

- la médiatisation d'une crise particulière et les risques de surenchère en termes d'annonces de dons;

- le problème des dons inappropriés (médicaments inutiles et reconstruction à la hâte de maisons présentant des défauts);

- les difficultés particulières de l'acheminement de l'aide dans certaines régions en situation fragile de postconflit (Sri Lanka et Aceh).

\subsubsection{Les autres crises humanitaires, des crises " oubliées "?}

Début janvier 2005, Médecins sans frontières (MSF) France a annoncé avoir récolté suffisamment de fonds pour les actions d'urgence qu'elle pouvait mener dans les pays touchés par le tsunami. L'ONG proposait aux donateurs qu'ils réorientent leur aide sur des crises oubliées comme en République démocratique du Congo, au Darfour ou au Niger, ou alors même qu'elle rembourse leurs dons à ceux qui le souhaitaient (moins de $1 \%$ du montant collecté a ainsi été remboursé $)^{18}$.

Très rapidement après la catastrophe du tsunami, des voix se sont élevées au sein de nombreuses ONG pour relever qu'il ne fallait pas que cette crise fasse oublier d'autres graves crises humanitaires, pour lesquelles il est plus difficile de récolter des fonds d'urgence ${ }^{19}$. Les Nations unies, le CICR et la DDC ont aussi rappelé à maintes reprises que d'autres causes très importantes et d'autres crises humanitaires mériteraient une attention plus grande de la part des donateurs. Pour donner quelques exemples de comparaison, début juillet 2005, la Chaîne du bonheur avait récolté 10 millions de francs pour les victimes du tremblement de terre à Bam (Iran, décembre 2003) et 1 million seulement en un mois pour aider les personnes déplacées à cause de la crise au Darfour (Soudan, deuxième campagne de récolte de fonds en juin 2005).

Beaucoup de professionnels d'ONG, d'organisations internationales et d'agences de coopération gouvernementales ont rappelé l'existence de «tsunamis silencieux» ou de «désastres sans visage» de par le monde: la situation au Darfour ou encore en République démocratique du Congo, en Ouganda ou dans la région des Grands Lacs, les famines, les victimes de la malaria, les conséquences dramatiques de l'invasion de criquets en Afrique... Le nombre de décès des personnes victimes de la pauvreté, de la faim et de maladies transmissibles dépasse chaque semaine celui causé par le tsunami.

L'ONU a aussi rappelé que les versements effectifs des gouvernements suite à des appels de fonds restent souvent largement en deçà des montants annoncés

17 «Herausforderungen nach den Hilfszusagen», Neue Zürcher Zeitung, 10. Januar 2005; «Hilsfwerke doppelt herausgefordert», Neue Zürcher Zeitung, 7. Januar 2005.

18 La Liberté, 6 janvier et 23 mai 2005.

19 Voir notamment InfoSud, Les désastres sans visage ont aussi besoin d'aide, 12 janvier 2005. 
sous les feux de l'actualité et que les gouvernements ne répondent que timidement à certains appels de fonds, les moyens financiers restant très insuffisants pour bien des causes pourtant importantes. Par exemple, seule une partie de l'aide promise par les pays donateurs suite au tremblement de terre à Bam, en Iran, en décembre 2003 a effectivement été versée après les annonces médiatiques. Plusieurs appels de fonds des Nations unies ont entraîné des promesses de dons très insuffisantes. Seul 1 million de dollars a ainsi été versé pour chacun des appels aux dons en 2005 concernant la République centrafricaine (47 millions de dollars demandés), la Côte d'Ivoire (40,5 millions de dollars demandés) et la Guinée (33 millions de dollars demandés ${ }^{20}$. Seuls 36,5 millions de dollars ont été versés pour la République démocratique du Congo au lieu des 738 millions demandés, et 311 millions de dollars promis au Pakistan (jusqu'en mars 2006) au lieu des 550 millions de dollars demandés.

L'Assemblée générale des Nations unies a créé le 15 décembre 2005 le Fonds central d'intervention d'urgence (Central Emergency Response Fund), alimenté par les pays membres, fonds qui doit devenir opérationnel en $2006^{21}$. De l'argent pourrait être ainsi utilisé rapidement lors d'une crise humanitaire, sans attendre les appels de fonds particuliers. En janvier 2006, 200 millions de dollars ont été accordés à ce fonds, dont 4 millions de francs de la Suisse, sur les 500 millions nécessaires au fonctionnement.

\section{$\square$ Séisme à Bam, Iran, 26 décembre 2003}

Le tremblement de terre en Iran, le 26 décembre 2003, avait détruit la ville de Bam, provoquant plus de 35'000 morts. Dix millions de francs avaient été récoltés par la Chaîne du bonheur. L'aide des ONG suisses s'est focalisée sur la reconstruction de maisons ou d'écoles, la réparation de canaux d'irrigation, la création de centres d'accueil et d'appui pour des enfants traumatisés et orphelins, et les soins aux handicapés.

\section{$\square$ Crise du Darfour (Soudan)}

La guerre civile dans le Sud-Soudan aurait entraîné la mort de plus de 2 millions de personnes et provoqué le déplacement forcé de plus de 4 millions de personnes. La DDC contribue depuis 1994 à l'Operation Lifeline Sudan, qui coordonne l'aide humanitaire dans les régions contrôlées par les forces non gouvernementales ${ }^{22}$. Après plusieurs années de pourparlers et de négociations de paix, un cessez-le-feu et un accord de paix sont intervenus en 2005, mettant un terme à un conflit interne qui déchirait le Soudan depuis les années 1950. Mais, depuis 2003, un nouveau conflit dans une province occidentale du Soudan, le Darfour, a provoqué un drame humanitaire et des déplacements importants de populations. L'armée a recouru à des tribus nomades locales pour attaquer la population civile du Darfour. Depuis l'automne 2003, la DDC finance des programmes du PAM, du HCR, du CICR et d'ONG au Darfour et au Tchad. Le Soudan fait partie des pays où l'engagement humanitaire de la Suisse est très fort (voir tableau 8).

20 «Mangelnde Mittel für Hilfe in vielen Katastrophengebieten», Neue Zürcher Zeitung, 28.-29. Januar 2006.

21 Voir le site de l'OCHA, <http://ochaonline.un.org > >more on the CERF.

22 L'action de la Suisse est résumée sur le site Internet de la DDC, <www.ddc.admin.ch > > pays >Soudan. 


\section{$\square$ Crise alimentaire au Niger et dans d'autres pays d'Afrique noire}

La pénurie alimentaire au Niger a été grave en 2005. Les Nations unies, le gouvernement du Niger, la DDC et des ONG ont attiré l'attention en automne 2004 déjà sur la baisse de la production agricole et le manque de céréales et de fourrage pour les animaux, avec le déficit le plus grave de l'histoire du Niger. Les dons internationaux alimentaires demandés par le PAM ont été insuffisants et les médias se sont mobilisés plus tard, en juillet-août 2005, notamment après les appels lancés par certaines ONG comme Oxfam et MSF.

Plusieurs facteurs expliquent cette crise. La sécheresse particulièrement forte en été 2004 (saison des pluies arrêtée prématurément) a entraîné une baisse importante des récoltes, et le pays a été fortement touché par l'invasion de criquets pèlerins. L'engrenage a été terrible, avec une perte de la valeur du bétail malade et une forte augmentation du prix des céréales. Les problèmes structurels dans l'agriculture du pays ont aggravé la crise: avancée du désert, irrigation insuffisante, manque de moyens pour les paysans pour acheter des intrants agricoles et moderniser leurs moyens de production rudimentaires, rendements agricoles faibles. Durant l'été 2005, sur une population totale de 12 millions d'habitants, 3,5 millions étaient touchés par la malnutrition.

Au problème de la sécheresse se sont ajoutés des dysfonctionnements dans les systèmes d'alerte précoces et de longs délais avant que les pays donateurs se mobilisent. Plusieurs ONG, dont Médecins sans frontières, ont accusé le gouvernement du Niger de tenter de minimiser l'ampleur de la crise en hiver 20042005 et d'avoir ainsi retardé la prise de conscience de son importance. La politique gouvernementale de refus de distribution ciblée gratuite de nourriture pour les plus pauvres (jusqu'en juillet 2005) a aussi suscité des critiques ${ }^{23}$. Le fait que les soins ne sont pas gratuits pour les pauvres a aussi ralenti la prise de conscience de l'ampleur de la catastrophe humanitaire, car les pauvres sont venus trop tard dans les centres de santé.

La famine ne touche pas uniquement le Niger; d'autres pays d'Afrique noire ont été sévèrement affectés en 2005, dont le Malawi ${ }^{24}$ et le Mali. La DDC a débloqué 1,5 million de francs pour le Niger et le Mali ${ }^{25}$, alors que les œuvres d'entraide ont décidé début août 2005 de fournir une aide de plus d'un million de francs pour les pays du Sahel frappés par la famine, avec pour le Niger la fourniture de céréales (millet), de fourrage et de semences ${ }^{26}$.

\section{$\square$ Tremblement de terre au Pakistan et en Inde}

Le violent tremblement de terre qui a secoué le Cachemire le 8 octobre 2005, au nord de l'Inde et surtout au Pakistan, a entraîné plus de 87'000 morts ${ }^{27}$. Dix mil-

23 «Le monde a été sourd. Niger, les raisons d'un désastre», Le Nouvel Observateur, 11 août 2005; «Niger, les origines d'un désastre annoncé », Le Temps, 12 août 2005 ; «Niger: combien de famines encore?», Le Monde, 19 août 2005.

24 «Famine à l'écart des caméras», Courrier international, no 778, 29 septembre 2005. Selon 1'OCHA, un tiers de la population du Malawi risque de ne pas avoir assez de nourriture jusqu'à la prochaine récolte de 2006.

25 DDC, Crise alimentaire en Afrique occidentale, communiqué de presse, 11 août 2005.

26 «Chronique d'une famine annoncée», Le Courrier, 22 juillet 2005; famine au Niger, Le Courrier, 3 août 2005 .

27 «Die Zahl der Bebenopfer in Pakistan nach oben korrigiert», Neue Zürcher Zeitung, 9 novembre 2005. 
lions de francs ont été débloqués par la DDC pour l'aide en Asie du Sud et quelque 45 experts du Corps suisse d'aide humanitaire ont été déployés sur le terrain. Deux millions de francs ont en outre été versés, notamment au PAM, pour affréter des hélicoptères de transport ${ }^{28}$. Plusieurs ONG suisses se sont aussi mobilisées.

\section{- Catastrophes naturelles en Amérique centrale,} dans le Sud des Etats-Unis et aux Caraïbes

Début octobre 2005, les fortes précipitations et l'ouragan Stan en Amérique centrale ont entraîné des glissements de terrain, principalement au Salvador, au Guatemala et dans le Sud du Mexique, avec des conséquences dramatiques sur l'agriculture (débuts de la récolte du café) et la destruction et l'ensevelissement de nombreuses maisons et de voies de communication. La DDC a envoyé de l'aide pour 800 '000 francs ${ }^{29}$. L'ouragan Katrina a été l'un des plus violents de l'histoire des Etats-Unis, provoquant une rupture de digues et de sévères inondations à La Nouvelle-Orléans. En 2005, le nombre de cyclones dévastateurs aux Caraïbes a été particulièrement élevé (une douzaine), ce qui a aussi sollicité des interventions nombreuses et coûteuses pour les organismes des Nations unies ${ }^{30}$.

\subsection{Conférence mondiale de Kobe}

sur la prévention des catastrophes naturelles (Japon, 18-22 janvier 2005)

Cette conférence internationale était agendée bien avant que ne survienne le tsunami, mais l'actualité a permis d'attirer une attention beaucoup plus grande de l'opinion politique et des gouvernements, avec une présence de plus de 4000 délégués gouvernementaux et non gouvernementaux. La conférence s'inscrivait dans la continuité de la Décennie internationale de la prévention des catastrophes naturelles, qui avait été proclamée par les Nations unies dans les années 1990.

La conférence a rappelé l'urgence de mettre en place et de coordonner au niveau planétaire des programmes de prévention et de préparation pour diminuer les risques et la vulnérabilité des populations exposées aux désastres naturels. L'augmentation du nombre de victimes humaines et l'ampleur des dégâts matériels depuis les années 1980 sont une source d'inquiétude croissante, avec notamment les conséquences des crues, des cyclones, des glissements de terrain, des tremblements de terre, des tsunamis et des épidémies.

La décision la plus concrète de la conférence a été de mettre sur pied un système d'alerte au tsunami dans les pays riverains de l'océan Indien, à l'image de ce qui existe dans le Pacifique.

La conférence a adopté un programme d'action fixant les mesures à prendre pour diminuer d'ici à 2015 l'impact des catastrophes naturelles sur les populations

28 DDC, Séisme en Asie du Sud. La Suisse augmente sa contribution à 2 millions, communiqué de presse, 11 octobre 2005.

29 DDC, Ouragan an Amérique centrale. La Suisse envoie de l'aide d'urgence, communiqué de presse, 7 octobre 2005; DDC, Ouragan «Stan» en Amérique centrale. La Suisse accrô̂t son aide, communiqué de presse, 12 octobre 2005.

30 «Die UNO am Rande der Überforderung», Neue Zürcher Zeitung, 12. Oktober 2005. Voir aussi: Fédération internationale des Sociétés de la Croix-Rouge et du Croissant-Rouge, Rapport sur les catastrophes dans le monde 2005. 
menacées ${ }^{31}$. Le cadre d'action décrit les tâches qui incombent aux Etats, aux organisations et institutions régionales et aux organisations internationales. Les Etats doivent prendre des mesures pour la prévention des risques de catastrophe, surveiller l'évolution de ces risques et améliorer l'alerte précoce. Les pays du Nord doivent soutenir la mise en œuvre du cadre d'action dans les pays en développement, intégrer des mesures de prévention dans les programmes d'aide au développement et accroître le financement de la reconstruction après des catastrophes.

La Suisse s'est fortement engagée dès les travaux de préparation de cette conférence. Elle a notamment conduit un atelier sur les dangers présents dans les régions de montagne. Si la conférence a été considérée généralement comme très positive, quelques critiques sont apparues, notamment sur le manque d'engagements concrets des donateurs et la suppression de la référence à la création de nouveaux mécanismes financiers pour aider les pays en développement à mettre sur pied des plans d'action nationaux de prévention des risques de catastrophes ${ }^{32}$.

\subsection{Nouvel emblème pour le Mouvement international de la Croix-Rouge et du Croissant-Rouge}

La conférence diplomatique qui s'est tenue à Genève du 5 au 8 décembre 2005 , avec des représentants de 144 Etats, a permis d'adopter un emblème additionnel pour le Mouvement international de la Croix-Rouge et du Croissant-Rouge. Depuis 1864, la croix rouge et, depuis 1929 , le croissant rouge sont les symboles universels du secours aux victimes des conflits armés et des catastrophes naturelles, comme signes visibles de la neutralité des missions humanitaires. L'adoption d'un nouvel emblème était nécessaire pour les Etats qui ne se reconnaissent dans aucun des deux emblèmes (notamment Israël), et les discussions duraient depuis 1999 mais avaient été ralenties par les événements en 2000 au Moyen-Orient. Le Protocole additionnel aux Conventions de Genève du 12 août 1949 relatif à l'adoption d'un signe distinctif additionnel (Protocole III) a été adopté le 8 décembre 2005 et permet ainsi d'ajouter l'emblème du cristal rouge, un dessin libre de connotation religieuse. La conférence internationale des 192 Etats parties aux Conventions de Genève et des 183 Sociétés nationales membres de la Fédération internationale des Sociétés de la Croix-Rouge et du Croissant-Rouge doit se réunir en 2006 pour changer les statuts du mouvement afin de prendre en compte la création du nouvel emblème ${ }^{33}$.

La Suisse s'était engagée activement dans la préparation de cette conférence, en tant que dépositaire des Conventions de Genève. Elle avait notamment, en novembre 2005, facilité un accord de reconnaissance respective entre le Croissant-Rouge palestinien et le Magen David Adom israélien. La conseillère fédérale Micheline Calmy-Rey aurait souhaité que l'adoption du nouvel emblème

31 Le document final adopté à la Conférence de Kobe s'intitule Cadre d'action de Hyogo pour 20052015: pour des nations et des collectivités résilientes face aux catastrophes, doc. A/CONF.206/L.2/Rev.1.

32 «Wenig Konkretes zum Katastrophenschutz. Abschluss der Uno-Konferenz in Kobe», Neue Zürcher Zeitung, 24. Januar 2005.

33 CICR, documentation sur le nouvel emblème et sur les conférences tenues depuis l'an 2000, disponible sur le site Internet du CICR, <www.icrc.org $>$. 
puisse se faire par consensus. Suite aux résistances de certains Etats, les discussions se sont prolongées, mais le protocole additionnel a finalement été adopté, par 98 oui, 27 non et 10 abstentions. Une majorité des deux tiers au minimum était nécessaire, soit 90 oui. La Syrie a saisi l'occasion de cette conférence pour réaffirmer sa souveraineté sur le Golan, annexé par Israël en 1981, en demandant - sans l'obtenir - que seul le Croissant-Rouge puisse opérer sur le Golan.

\section{SOURCES}

CAD (OCDE), Examen par les pairs. Suisse. 2005, Paris, OCDE, 2005, 126 p., en particulier annexe C, «Evaluation de l'aide humanitaire de la Suisse», pp. 105-115.

Dayton-Johnson J., Natural Disasters and Adaptive Capacity, OECD Development Centre Working Paper, no 237, Paris, OCDE, August 2004, 40 p.

Fédération internationale des Sociétés de la Croix-Rouge et du Croissant-Rouge, Rapport sur les catastrophes dans le monde 2005.

DDC, Journée annuelle de l'Aide humanitaire et du CSA «Les catastrophes naturelles», Berne, documentation de presse, $1^{\text {er }}$ avril 2005.

DDC/seco, Rapport annuel de la coopération internationale de la Suisse 2004, Berne, 2005, 24 p.

Chaîne du bonheur, Rapport annuel 2004, 2005, 50 p.

Chaîne du bonheur, Tsunami. Un an après. Les projets de la Chaîne du bonheur, décembre 2005, 24 p.

Institut international du développement durable (IIDD), Résumé de la Conférence mondiale pour la prévention des catastrophes: 18-22 janvier 2005, Bulletin des Négociations de la Terre, vol. 26, $\mathrm{n}^{\circ} 9$, 24 janvier 2005, téléchargeable sur Internet: <www.iisd.ca/isdr/wcdr1>.

Schroeder R., Varga C., van Dok G., Les défis humanitaires. Les dimensions politiques de l'aide d'urgence, Caritas Suisse et Caritas Luxembourg, 2005, 164 p.

Terre des Hommes Lausanne, Terre des hommes, dossier sur l'aide d'urgence, mars 2005.

Werly R., Tsunami. La vérité humanitaire, Paris, Editions du Jubilé, 2005.

Presse

Le Temps, aide de la Suisse en Thaillande suite au tsunami, 9 avril 2005; «Cachemire: les limites de 1'humanitaire», 13 octobre 2005.

Neue Zürcher Zeitung, «Erfolgreiche schweizerische Hilfe in Thailand», 26.-27. März 2005; «Mehr Prävention gegen Naturkatastrophen. Konsequenzen des Tsunami für die humanitäre Hilfe», 2.-3. April 2005.

Revue médicale suisse, «Le don: un "basic instinct"?», n 2, 2005; «Clarifier la générosité», $\mathrm{n}^{\circ}$ 19, 2005.

\section{SITES INTERNET}

Bureau de la coordination des affaires humanitaires des Nations unies (OCHA): <http://ochaonline.un.org $>$. Comité international de la Croix-Rouge (CICR) : <www.icrc.org $>$.

Direction du développement et de la coopération (DDC) : <www.ddc.admin.ch>> >aide humanitaire.

Chaîne du bonheur: <www.bonheur.ch>. 